\title{
Mastering your Fellowship
}

\author{
Klaus B von Pressentin, ${ }^{1 *}$ Mergan Naidoo, ${ }^{2}$ Langalibalele Honey Mabuza ${ }^{3}$ \\ ${ }^{1}$ Family Medicine and Primary Care, Stellenbosch University \\ ${ }^{2}$ Family Medicine, University of KwaZulu-Natal \\ ${ }^{3}$ Family Medicine and Primary Health Care, Sefako Makgatho Health Sciences University \\ *Corresponding author, email: kvonpressentin@sun.ac.za
}

\section{Abstract}

The series, "Mastering your Fellowship", provides examples of the question format encountered in the written examination, Part A of the FCFP(SA) examination. The series aims to help Family Medicine registrars prepare for this examination. Model answers are available online.

Keywords: FCFP(SA) examination, Family Medicine registrars

\section{Introduction}

This section in the South African Family Practice journal is aimed at helping registrars prepare for the FCFP (SA) Part A examination (Fellowship of the College of Family Physicians) and will provide examples of the question formats encountered in the written examination: Multiple Choice Question (MCQ) and/or Extended Matching Question (EMQ), Modified Essay Question (MEQ) and Critical Reading paper (evidence-based medicine). Each of these question types is presented according to a theme. The MCQs will be based on the ten clinical domains of family medicine, the MEQs will be aligned with the five national unit standards and the critical reading section will include evidence-based medicine and primary care research methods. We suggest that you attempt answering the questions (by yourself or with peers/ tutors), before finding the model answers online: http://www. safpj.co.za/.

Please visit the Colleges of Medicine website for guidelines on the Fellowship examination: http://www.collegemedsa.ac.za/ view_exam.aspx?examid $=102$

We are keen to hear about how this series is assisting registrars and their supervisors in preparing for the FCFP (SA) examination. Please email us your feedback and suggestions.

1. EMQ (extended matching question) Theme: Abdominal pain

1.1 A 21-year-old man presents with severe abdominal pain in his right flank, which radiates to his back and right inguinal area. He does not have a fever and his BP is 160/100 with a pulse rate of $110 /$ minute. His abdomen is moderately tender. His urine dipstick test shows $1+$ blood.

1.2 A 50-year-old woman presents with pain in the lower left quadrant worsening over the last 36 hours. She has felt febrile and her appetite is decreased. She reports a change in bowel habit with intermittent constipation and flatulence over several years. Her temperature is $38.5^{\circ} \mathrm{C}$, BP $140 / 90$ and pulse 100 /minute. Her abdomen is soft with voluntary guarding in the lower left quadrant with hypoactive bowel sounds. Rectal examination is unremarkable. Laboratory studies show a leucocyte count of $15.3 \times 10^{3} / \mathrm{mm}^{3}$.

1.3 A 47-year-old woman presents with severe epigastric pain radiating to her back. The pain worsened after eating breakfast and she vomited brown fluid. The pain has been increasing in severity and has now become constant. She has a vague history of abdominal complaints and has been treated for abdominal colic previously. She also has hypertension, hypothyroidism and diabetes mellitus. On examination she is an obese woman in mild distress. Her vital signs are normal with a temperature of $37.3^{\circ} \mathrm{C}$. Her abdomen is diffusely tender with guarding.

1.4 A 60-year-old man presents with severe abdominal pain, nausea and vomiting. He describes the pain as constant and excruciating. He had one episode of slightly loose stools when the pain started early this morning, but has not passed any stools since. The pain is worse after eating. He is a smoker with a 40 pack-year history and has a history of hypertension for which he takes hydrochlorothiazide. On examination he has no fever, his BP is $110 / 60$ and a pulse of 110 /minute. His abdomen appears slightly distended, but is soft with mild diffuse tenderness on palpation.

1.5 A 50-year-old woman presents with abdominal pain which started in the epigastric region, but is now felt in the right upper quadrant. She describes the pain as a constant pain. She has a vague history of similar abdominal complaints. She is also nauseous. On examination she is an obese woman who has a temperature of $38.3^{\circ} \mathrm{C}$, BP of $130 / 80$ and a pulse rate of 100 /minute. Her abdomen is tender in the right upper quadrant with voluntary guarding. Urine dipstick test is normal, full blood count shows leucocytosis.

For each of the patient scenarios listed ABOVE choose the best option from the list BELOW. Each option may be used once, more than once, or not at all.
A. Intestinal obstruction
B. Diverticulitis 

C. Ovarian carcinoma
D. Appendicitis
E. Acute cholecystitis
F. Pancreatitis
G. Hepatitis
H. Peptic ulcer disease
I. Dissecting aortic aneurysm
J. Renal calculi
K. Irritable bowel syndrome
L. Acute mesenteric vascular insufficiency

2. SAQ (short answer question): the family physician's role as a family-oriented care provider

You have been the family physician in a Community Health Centre $(\mathrm{CHC})$ for the past year. The next patient to enter your consultation room is John, a 9-year-old boy and his mother. $\mathrm{He}$ is attending a local, mainstream primary school and the school psychologist arranged for him to see you at the $\mathrm{CHC}$, as his teacher reported him to be ill-disciplined in the classroom, not obeying instructions, not paying attention during lessons, fighting with other children in the school and not progressing well academically.

John lives with his parents and three siblings (three girls Mary, Anne and Jane aged 5, 11 and 13 years respectively). His mother, Kate (aged 41 years), recently lost her younger sister, Sue (aged 36 years), due to HIV-related complications; Sue's 18-month old daughter (Kerry) is now living in this household and is stable on her antiretroviral therapy. The patient's father (aged 44 years) is a welder by profession and has a stable income. John's parents are generally healthy, except for his mother having well-controlled asthma. The eldest daughter has a close relationship with their mother and helps with the adopted toddler after school. This 9-year-old boy had regular altercations with his father, Peter, and eldest sister, especially as he was caught stealing money from his father. His maternal grandmother, Pam (aged 71 years, wellcontrolled on anti-hypertensives) stays with them in the house, and tends to dominate over Kate. His maternal grandfather, Jack, died in 2009 after suffering a myocardial infarct. His parents are married and his grandparents were married. Kerry's father's name and whereabouts are unknown.

2.1 Draw a genogram in which you highlight the relationships within this household. (5 marks)

2.2 The school teacher recommends that your start him on methylphenidate. Briefly outline your response to this recommendation, by addressing the following aspects.

2.2.1 What key areas should you focus on during your consultation with the child and his mother? (5 marks)

2.2.2Discuss your approach to making a decision on whether methylphenidate is indicated, including the differential diagnosis and management options to consider for this patient. (5 marks)
2.3 You decide to arrange a family conference. Briefly outline the steps for conducting a family conference with this family in mind. (5 marks)

\section{Critical appraisal of research}

Please answer the questions related to the following article:

Essel V, Van Vuuren U, De Sa A, et al. Auditing chronic disease care: Does it make a difference? Afr J Prm Health Care Fam Med. 2015;7(1), Art. \#753, 7 pages. http://dx.doi.org/10.4102/phcfm. v7i1.753. (Total 50 marks)

\section{Introduction (9 marks)}

3.1 What study design was used in this study?

3.2 How would you define the study design used?

3.3 List three (3) advantages and three (3) disadvantages of this design.

\section{Methods (12 marks)}

3.4 The authors stated that through this study they aimed at assessing the effectiveness of clinical audits done for NCD care. Briefly state how they achieved this aim?

3.5 Explain the sampling method used in this study?

3.6 Why was the median more appropriate than the mean in the percentile analysis?

\section{Results (14 marks)}

3.7 What is the overall message conveyed by Table 3 ?

3.8 Study Table 4 and interpret the following:

3.8.1 The odds ratio of $0.32[95 \% \mathrm{Cl}(0.28-0.37)]$ with respect
to the rural districts.

3.8.2The odds ratio of 0.52 [ $95 \% \mathrm{Cl}(0.24-1.10)]$ in the recording of asthma.

3.8.3The odds ratio of 3.47 [95\% $\mathrm{Cl}(1.90-6.33)]$ in the recording of COPD.

\section{Study strengths and limitations (8 marks)}

3.9 List the limitations (weaknesses) of this study.

\section{Take-home message (7 marks)}

3.10 What has this study added to your knowledge on the topic?

3.11 Mention five (5) points you would consider before accepting the findings of a study for implementation in your setting.

\section{Model answers to questions}

\section{Question 1}

\section{Short answer:}

$\begin{array}{ll}1.1 & \text { J } \\ 1.2 & \text { B } \\ 1.3 & \text { F } \\ 1.4 & \text { L } \\ 1.5 & \text { E }\end{array}$




\section{Long answer:}

As a family physician working the primary care/district hospital environment, it is not always possible to have access to many of the investigations available at higher levels of care. This is when one's clinical acumen and reasoning plays a big role in determining the most likely clinical pathway that the patient should follow. The above scenarios describe surgical causes for abdominal pain that can alert the astute clinician on causation.

Scenario 1.1 describes the typical presentation of a patient who presents with acute renal colic. This pain is typically severe with radiation to the flank, back or the groin. Nausea and vomiting are common symptoms and haematuria on dipsticks is not always present.

Scenario 1.2 describes the typical picture of diverticulitis with left lower quadrant pain, change in bowel habits with constipation and flatulence being common presenting symptoms. Localised tenderness and fever suggests an inflammatory cause of the pain.

Scenario 1.3 is more difficult to piece together. This patient presents with recurrent abdominal pain and nausea and vomiting. She also has obesity and chronic medical conditions. The history would suggest that she is a patient who has risk factors for gallstones which is one of the causes of pancreatitis. A serum amylase and lipase will assist with the diagnosis.

Scenario 1.4 describes a picture where the pain is disproportionate with the clinical examination. The pain is usually worse post- prandial, typically occurring 10-20 minutes after eating and sometimes lasting for hours ("abdominal angina"). The patient also has risk factors for arterial thrombosis which warrants future investigation and management. Acute mesenteric arterial thrombosis typically occurs in patients who are prone to atherosclerosis. The pathophysiology is similar to that of an acute coronary syndrome with plague rupture and thrombosis.

Scenario 1.5 describes the typical presentation of acute cholecystitis. Pain often starts in the epigastrium and then radiates to the right upper quadrant. The pain is typically colicky in nature and nausea, vomiting, bloating and fever are frequent findings. Liver function tests may reveal features of abnormally raised enzymes suggesting hepatitis or obstruction. Diagnosis is usually confirmed with ultrasound assessment.

\section{Further reading:}

- Chapter 7: An approach to assessing common symptoms. In: Mash B, editor. Handbook of Family Medicine. 3rd ed. Cape Town: Oxford University Press Southern Africa; 2011: p. 203-7.

- Medscape. (2017). Medscape Mobile (v4.1.1) [Mobile application software]. Retrieved from: http://www.medscape. com/public/mobileapp/features

\section{Question 2}

\section{Model answer:}

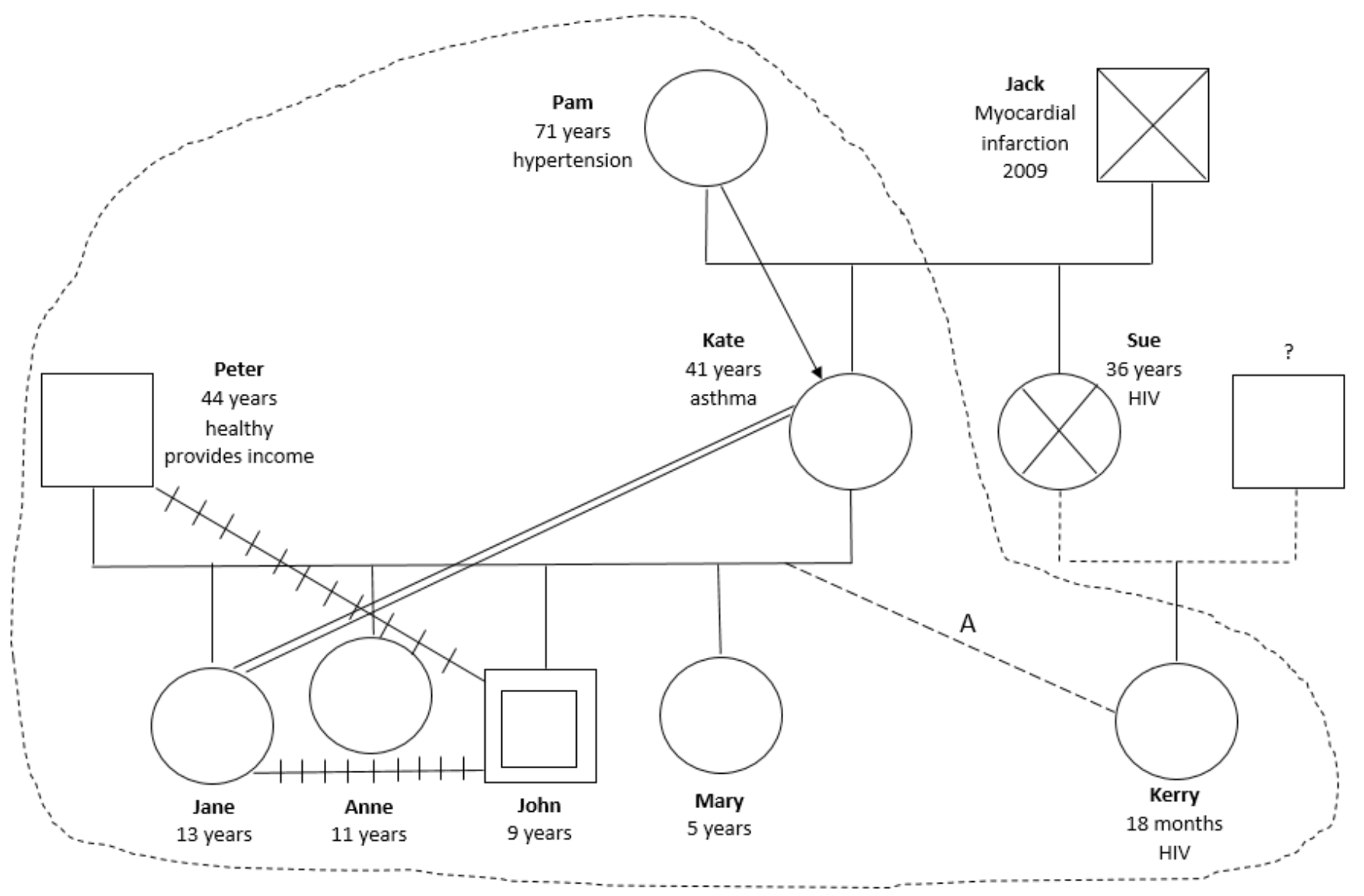

Figure 1: Genogram (Question 2) 


\subsection{Draw a genogram in which you highlight the relationships within this household. (5 marks)}

The genogram in Figure 1 provides a pictorial representation of the three generations of family. This useful tool allows one to gather information about the family's relationships systematically. The dashed line defines the household members. This genogram also depicts the relationships between family members in terms of closeness, conflict and dominance. The adoption of the daughter of the mother's sister is also shown.

\subsection{The school teacher recommends that you start him on methylphenidate. Briefly outline your response to this recommendation, by addressing the following aspects.}

\subsubsection{What key areas should you focus on during your consultation with the child and his mother? (5 marks)}

- Remember that this is a paediatric consultation: start with a proper history and take care to focus the interview on the child, his context and his family.

- Allow him and his mother to share their story by creating a relaxed and open atmosphere.

- Attempt to understand their meaning, motivation and thoughts regarding the dynamics and relationships at home and at school (here it is relevant to focus on these two contexts).

- Obtain a thorough history on the antenatal aspects (exposure to toxins or alcohol), enquire about any perinatal problems (delivery complications, APGAR scores, neonatal problems) and review his developmental milestones (especially the first twoyears of life).

- Review the notes of previous consultations and investigations in his health records (this may not be the first contact with the $\mathrm{CHC}$ ). Take care, however, not to accept any diagnostic labels assigned previously.

- Conduct a complete clinical examination, with a special focus on the eyes (visual acuity), ears (hearing), speech and neuro-developmental aspects.

2.2.2 Discuss your approach to making a decision on whether methylphenidate is indicated, including the differential diagnosis and management options to consider for this patient. (5 marks)

- Methylphenidate is indicated for attention-deficit/ hyperactivity disorder (ADHD), a common neurobehavioural disorder which presents with the core symptoms of developmentally inappropriate levels of inattention, impulsivity and hyperactivity. A diagnosis of ADHD is made clinically, based on behavioural symptoms, according to the Diagnostic and Statistical Manual of Mental Disorders, Fifth Edition (DSM-5) criteria. The symptoms need to be assessed according to the patient's age and developmental level, as some of them may be normal at different stages of development.

- Before considering a diagnosis of ADHD and initiating methylphenidate, rule out possible differential diagnoses. Co-morbidities are more the rule than the exception, with $80 \%$ of patients having a co- morbid psychiatric condition, which may include other neurodevelopmental disorders like learning and communication disorders or autism; externalising disorders, such as oppositional defiant disorder or conduct disorder; or internalising disorders, such as anxiety and depression. Also consider intellectual or cognitive disability, such as foetal alcohol spectrum disorder.

- Visualise this child and his family in context (the genogram is very helpful in this regard). Is this a "signal child", whose behavioural or presenting problems are pointers or signals to problems experienced in the household?

- Consider the stages of the family life cycle and development: is there any link with the duration of his behavioural issues and the introduction of the adopted child into the household?

- Doyou have enough information or doyou require input from the rest of the multidisciplinary team? Information from more than one setting, e.g. home and school, is needed when conducting an evaluation for ADHD. It is recommended that you obtain reports/assessments from the teacher/school principal, school/educational psychologist, occupational therapist, speech therapist, audiologist, optometrist and social worker, as indicated. Consider referring him also for assessment by a child psychiatrist or psychologist (clinical or educational), and/or paediatrician, especially if you are considering psychiatric co-morbidities.

- Long-term treatment is needed for ADHD as it is a chronic condition. Treatment needs to be adjusted according to the patient's age, stage of development and the side-effects experienced. The two pillars of treatment are medication and different forms of behavioural therapy and/or other forms of psychosocial intervention.

- In conclusion, your initial response to the school teacher would be a respectful answer indicating that you require more information before initiating methylphenidate. You could share your strategy and ask the teacher to help obtain the reports/assessments from the education psychologist and school principal, as indicated.

\subsection{You decide to arrange a family conference as part of} the diagnostic and therapeutic plan. Briefly outline the steps for conducting a family conference with this family in mind. (5 marks)

- Start by reviewing the indication for the family conference. This family is going through a transition point, with the adoption of the toddler. The 9-year-old son's management plan has implications for this family.

- The success of this family conference depends on adequate planning. Preconference tasks to consider for this family, include:

- Setting the stage: discuss the potential value of the conference with the patient's mother. Involve her in deciding which family members should attend. It may be necessary to invite only the adults in the household. 
- Review the genogram (Figure 1) and develop hypotheses on how this family is functioning. The formulation of a hypothesis is crucial as it places the family physician in the active position of identifying relational patterns (the dominance of the maternal grandmother, the friction between the son and his father, possible tension between the patient's parents).

- Develop a strategy for conducting the conference (here you may wish to obtain more information on possible differential diagnoses and potential triggers for the child's conduct within the family context).

- Conference tasks should follow a clear structure:

- Socialise: greet the family, introduce yourself and make everyone feel comfortable. Observe how the different family members choose their seating arrangements, as this may highlight underlying relational preferences.

- Set the goals: clarify the reason for the meeting (for example, discussing the patient's behaviour problems and how they affect the family context); elicit each person's ideas of the problems in the family. Give equal opportunities for inputs in order to maintain the perception of neutrality in the eyes of the family.

- Discuss the impact of problems or issues - remain focused on the priority goals. Solicit each family member's view of the issues and how they impact their lives. Encourage questions. Enquiry about past experience of dealing with similar problems.

- Identify resources with the family unit, as well as medical and community resources. Enable the family to have realistic expectations of what can be provided within your resource-constrained environment.

- Establish a plan and clarify each person's role. How will they support each other and the patient during the diagnostic and therapeutic stages of his management for his chronic condition?

- Conclude by reiterating the family strengths, moving towards constructive solutions and reinforcing the sense of unity in the family (or accepting a tolerance for uncomfortable feelings, including anger, around the child's disruptive behaviour).

- Post-conference tasks for the family physician include revising the genogram, revising the preconference hypotheses and writing a conference report, which may be included in the patient's medical notes and shared with members of the multidisciplinary team, as indicated.

\section{Further reading:}

- Blitz J. Chapter 148: How to assess family structure and resources. In: Mash B, Blitz J, editors. South African Family Practice Manual. 3rd ed. Cape Town: Van Schaik; 2015: p. 486-7.

- Blitz J. Chapter 150: How to hold a family conference. In: Mash B, Blitz J, editors. South African Family Practice Manual. 3rd ed. Cape Town: Van Schaik; 2015: p. 490-2.

- Sparks B. Chapter 142: How to do a paediatric consultation. In: Mash B, Blitz J, editors. South African Family Practice Manual. 3rd ed. Cape Town: Van Schaik; 2015: p. 467-8.

- Bresick G. Chapter 4: Family-orientated primary care. In: Mash
B, editor. Handbook of Family Medicine. 3rd ed. Cape Town: Oxford University Press Southern Africa; 2011: p. 97-127.

- Du Plessis AM. Attention-deficit/hyperactivity disorder: review. South African Family Practice. 1 May 2015;57(3):24-8.

\section{Question 3}

\section{Model answer:}

\subsection{What study design was used in this study?}

Descriptive survey (although the authors called it a crosssectional study)

\section{Note (not part of model answer):}

One needs to be clear about the difference between a survey which is purely descriptive and a cross-sectional observational study which analyses and compares groups.

This study actually presents a number of sequential annual descriptive surveys (without trying to analyse them comparatively; they are just presented and the reader can see changes). The participating facilities differed between each survey making it difficult to analyse paired groups over time.

\subsection{How would you define the study design used?}

A survey is a study which describes data and characteristics about the population or phenomenon being studied. However, it does not answer questions about e.g.: how/when/why the characteristics occurred, which is done under analytic research.

\subsection{1 List three (3) advantages and three (3)} disadvantages of this design.

Table 1: Advantages and disadvantages of descriptive surveys

\begin{tabular}{|c|c|}
\hline Advantages & Disadvantages \\
\hline $\begin{array}{l}\text { - Good for descriptive } \\
\text { analyses. } \\
\text { - Is not costly to perform. } \\
\text { - Relatively quick and easy to } \\
\text { conduct (no long periods of } \\
\text { follow-up). } \\
\text { - Data on all variables is only } \\
\text { collected once. } \\
\text { - Good for generating } \\
\text { hypotheses. } \\
\text { - Able to measure prevalence } \\
\text { for all factors under } \\
\text { investigation. } \\
\text { - Multiple outcomes and } \\
\text { exposures can be studied. } \\
\text { - Used to assess the burden } \\
\text { of disease in a specified } \\
\text { population to enable } \\
\text { planning and allocating } \\
\text { health resources. }\end{array}$ & $\begin{array}{l}\text { - Unable to measure incidence. } \\
\text { - Cannot be used to analyse } \\
\text { behaviour/outcome over a } \\
\text { period to time. } \\
\text { - The timing of the snapshot } \\
\text { is not guaranteed to be } \\
\text { representative. } \\
\text { - Not suitable for studying rare } \\
\text { diseases or diseases with a } \\
\text { short duration. } \\
\text { - Susceptible to bias due to } \\
\text { low response. }\end{array}$ \\
\hline
\end{tabular}

\section{Methods (12 marks)}

3.4 The authors stated that through this study they aimed at assessing the effectiveness of clinical audits done for NCD care. Briefly state how they achieved this aim?

- Results were compared between years (for "2012 old" and "2012 new") and for structural, process and outcome criteria for specific diseases - diabetes, hypertension, etc.

- The implication was that these audits would be used as part of QIPs to improve quality of care. 
- Data was aggregated for the province as a whole and not by district.

\subsection{Explain the sampling method used in this study?}

In 2012, all the six Western Cape Province health districts participated in the audit, collectively listing 326 PHC facilities providing services to patients with NCDs. (2)

The audit itself was done in 2012 from data collected over four years (2009-2012) yielding 129 facilities by 2012. (1)

Of the $326 \mathrm{PHC}$ facilities, 129 were selected for auditing (72 from the "2012 old" districts and 57 from the "2012 new" districts). (1)

The selection of the facilities was based on the feasibility to perform the audit. (1)

Furthermore, a standardised chronic disease audit tool was used to evaluate two components: (a) the facility's process and equipment availability and (b) a folder review of five chronic conditions. (1)

Ten folders per chronic condition were sampled through a random systematic sampling method. (1)

The total number of folders audited was 6450. (1)

\subsection{Why was the median more appropriate than the mean in the percentile analysis?}

The data were not normally distributed and hence median percentages were used to present the results.

\section{Results (14 marks)}

\subsection{What is the overall message conveyed by Table 3? (5)}

The difference between the "2012 old" and "2012 new" is statistically significant across all five chronic disease indicators, all in favour of "2012 old" districts ( $p<0.05)$. The implication is that there was an improvement in the districts that began auditing from 2009 to 2012 compared to those that had started recently in 2011 and 2012.

\subsection{Study Table 4, and interpret the following:}

3.8.1 The odds ratio of $0.32[95 \% \mathrm{Cl}(0.28-0.37)]$ with respect to the rural districts.

Across all five chronic disease indicators, rural districts were 0.68 times less likely to record clinical processes compared to the Metro district.

\subsubsection{The odds ratio of 0.52 [95\% $\mathrm{Cl}(0.24-1.10)]$ in the recording of asthma.

Folders on asthma were 0.48 times less likely to have recorded clinical processes, compared to diabetes (the reference).

\subsubsection{The odds ratio of 3.47 [95\% $\mathrm{Cl}(1.90-6.33)]$ in the recording of COPD.

Folders on COPD were 3.47 times more likely to have recorded clinical processes, compared to diabetes (the reference).

\section{Study strengths and limitations (8 marks)}

3.9 List the limitations (weaknesses) of this study.
Reporting bias as the audit was done internally in each facility without internal and external validation. (2)

Selection bias as facility selection was based on feasibility to perform the audit (the criteria of which feasibility were not outlined). This implies that there were those excluded. (2)

Data to differentiate clinics participating in the audit for the first time from those who had audited previously were only available in 2012. (2)

The change in number of clinics participating was a confounding factor as changes in the audit results could be due to this rather than improvements in quality of care. (2)

Take-home message (7 marks)

\subsection{What has this study added to your knowledge on the} topic?

Clinical audits are useful in creating awareness about standards of care and for facilities to use their own data to identify areas that require improvement. Furthermore, clinical audits are preferable to routinely collected data as the data collected is of higher quality to bring about quality improvement.

\subsection{Mention five (5) points you would consider before} accepting the findings of a study for implementation in your setting.

- Is the topic relevant and/or important to my practice (relevance)?

- Are the findings valid (scientific rigour of the study methodology)?

- Are there similarities of the study population to my own practice (applicability)?

- If I choose to implement a change in my practice as a result of this study, will the implementation be acceptable to my population? (acceptability)

- Do I have the resources to implement the change (feasibility)?

\section{Further reading:}

- Mash B, Ogunbanjo GA. African Primary Care Research: Quantitative analysis and presentation of results. African Journal of Primary Health Care \& Family Medicine. 2014;6(1).

- Pather M. Continuing professional development. In Mash B (Ed) Handbook of Family Medicine (3rd ed). Cape Town: Oxford University Press, 2011: p. 406-29.

- Denscombe M. The good research guide: for small-scale social research projects. McGraw-Hill Education (UK); 1 Aug 2014.

- Szumilas M. Explaining odds ratios. J Can Acad Child Adolesc Psychiatry. 3 Aug 2010 (Accessed 2017 February 20);19:227. Available at: https://www.ncbi.nlm.nih.gov/pmc/articles/ PMC2938757/.

- Howick J. Introduction to study design. (Accessed 19 February 2017). Available at: http://www.cebm.net/wp-content/ uploads/2014/06/CEBM-study-design-april-2013.pdf. See also: http://www.cebm.net/study-designs/.

\section{Acknowledgements:}

- Prof Bob Mash for his help with reviewing the manuscript. 